\title{
Topological analysis of chaotic solution of a three-element memristive circuit
}

\author{
Jean-Marc Ginoux, \\ Laboratoire Protee, I.U.T. de Toulon, \\ Université du Sud, BP 20132 F-83957 La Garde Cedex, France, \\ Christophe Letellier, \\ CORIA UMR 6614, Université de Rouen, \\ BP 12 F-76801 Saint-Etienne du Rouvray cedex, France, \\ Leon O. Chua, \\ Electronics Research Laboratory \\ and Department of Electrical Engineering and Computer Sciences, \\ University of California, Berkeley, CA 94720
}

July 17,2021

\begin{abstract}
The simplest electronic circuit with a memristor was recently proposed. Chaotic attractors solution to this memristive circuit are topologically characterized and compared to Rössler-like attrators.
\end{abstract}

Keywords: Memristor; electronic circuit; chaos; topological analysis.

\section{Introduction}

In classical electronics, there are three passive circuit elements: the resistor, the capacitor and the inductor. In 1971, Leon Chua introduced a fourth "missing" element which he named a memristor - for memory resistor by using symmetry arguments Chua, 1971. Chua also derived the properties of this element. However, it is only in 2008 that Strukov and co-workers Strukov et al, 2008 found a memristance arising in a nanoscale system in which solid-state electronic and ionic transport are coupled under an external bias voltage. In 1976 Chua and Kang generalized the memristor to a broader class of nonlinear dynamical systems they called memristive systems Chua, 1976. Few chaotic memristive electronic circuits were already 
proposed Itoh \& Chua, 2008 and Muthuswamy \& Kakate, 2010 but they were four-dimensional. It is only recently that a three-dimensional system was proposed to describe a memristive circuit Muthuswamy \& Chua, 2010. This 3D system is the simplest three-element electronic circuit producing chaotic behaviors since it is only made of two linear passive energy-storage elements, and an active memristive device Chua, 1976. Such a simple electronic circuit is not an algebraically minimal system for which only five terms in the three right members of the set of ordinary differential equations are allowed [Zhang \& Heidel, 1997]. The system here studied has five linear terms and two nonlinear terms. The fact that this simple memristive circuit is not a minimal system is an advantage since very often, minimal systems have very tiny parameter domains associated with chaotic regimes and small attraction basin. As a consequence, the simple memristive circuit has a quite large attraction basin and a quite large domain of its parameter space over which the system is chaotic.

In this letter, we will perform a topological analysis of chaotic attractors of this memristive circuit. The subsequent part of this letter is organized as follows. Section 2 is devoted to the governing equations and their geometric interpretation in terms of flow curvature manifold. In section 3 the topological anlaysis in terms of branched manifold - or template - is presented. Section 4 gives some concluding remarks.

\section{The governing equations and their geometric in- terpretation}

\subsection{The set of differential equations}

The simplest electronic circuit producing chaotic attractors was proposed by Muthuswamy \& Chua, 2010. The corresponding block diagram is shown in Fig. 1. It is made of a linear passive inductor, a linear passive capacitor and a nonlinear active memristor. This electronic circuit can be described by the set of three differential equations

$$
\left\{\begin{array}{l}
\dot{x}=y \\
\dot{y}=-\frac{x}{3}+\frac{y}{2}-\frac{y z^{2}}{2} \\
\dot{z}=y-\alpha z-y z
\end{array} .\right.
$$


It is written here in a slighly modified form since the third equation is not 1

$$
\dot{z}=-y(1-z)-\alpha z
$$

as in Muthuswamy \& Chua, 2010 but rather

$$
\dot{z}=y(1-z)-\alpha z
$$

The nonlinearity is thus inverted. As a consequence, the orientation of the attractor in the $x-y$ plane is rotated by $\pi$ as easily checked in Fig. 2, There is no difference observed in the topology of these two attractors. This system has a single fixed point located at the origin of the phase space.

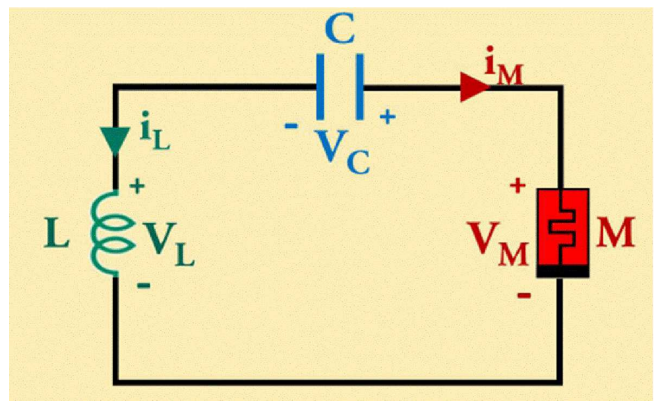

Figure 1: Block diagram of the simplest electronic circuit producing chaotic behavior.

\subsection{Flow curvature manifold}

Any trajectory solution to the dynamical system (1) and denoted $\boldsymbol{X}(t)$ describes the motion of a point $M$ in the phase space $\mathbb{R}^{3}(\boldsymbol{X})$ whose position depends on time $t$. This curve can also be defined by its parametric representation

$$
\left\{\begin{array}{l}
x=F_{x}(t) \\
y=F_{y}(t) \\
z=F_{z}(t)
\end{array}\right.
$$

where $F_{i}$ are the right members of the governing equations (11) that are assumed to be continuous, $C^{\infty}$ functions. The curvature $\kappa_{1}$ of any solution

\footnotetext{
${ }^{1}$ We picked a slightly different memristor characteristic to demonstrate the simplest three-element chaotic circuit in Fig. 1 is robust with respect to the choice of the memristor nonlinearity.
} 


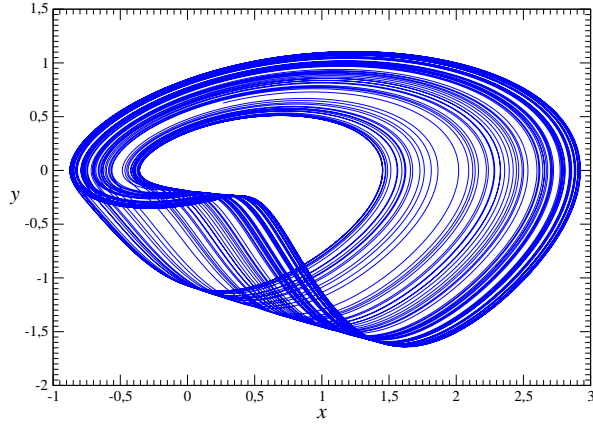

(a) With third equation (3)

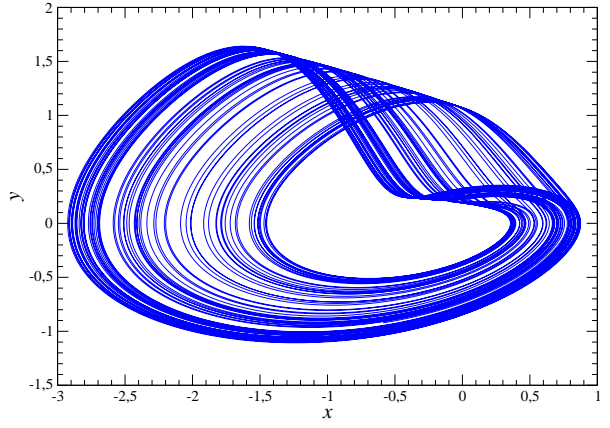

(b) With third equation (2)

Figure 2: Chaotic attractor solution to the minimal electronic circuit with a memristor. Parameter value: $\alpha=0.98$.

to system (11) is defined as

$$
\kappa_{1}=\frac{\operatorname{Det}(\dot{\boldsymbol{X}}, \ddot{\boldsymbol{X}})}{\|\dot{\boldsymbol{X}}\|^{3}}
$$

Curvature measures the deviation of the curve from a straight line in the neighborhood of any of its points. In a similar way, the torsion $\kappa_{2}$ is defined as

$$
\kappa_{2}=\frac{\operatorname{Det}(\dot{\boldsymbol{X}}, \ddot{\boldsymbol{X}}, \dddot{\boldsymbol{X}})}{\|\dot{\boldsymbol{X}} \wedge \ddot{\boldsymbol{X}}\|^{2}} .
$$

The torsion expresses the departure between the solution to system (11) and its projection into a place locally tangent to it.

It is known since Poincaré that the fixed points of a dynamical system provides some information on the structure of the solution to that system into the corresponding phase space. Fixed points belong to the zero-dimensional invariat set of the dynamical system. It is also known that fixed points do not provide all information needed to fully understand the structure of the trajectories in the phase space. Good examples of such situation include dynamical systems producing chaotic attractor structured around a single fixed point. Few of them may be found in the collection of quadratic systems proposed by Sprott, 1994. 
To extract additional information, nullclines were sometimes used as in Thomas \& Kaufman, 2001. But this is found only in the works made in the context of Fluid Mechanics Roth \& Peikert, 1998 where one-dimensional invariant sets were investigated as connecting fixed points (when there are more than one) and structuring the flow. One-dimensional invariant set can be defined according to the following theorem Gilmore et al, 2010.

Theorem 1. The one-dimensional invariant set of a three-dimensional dynamical system is defined as the location of the points where the velocity vector field is co-linear to the acceleration vector field, that is, to the location of the points where

$$
\kappa_{1}=0 \Leftrightarrow \operatorname{Det}(\dot{\boldsymbol{X}}, \ddot{\boldsymbol{X}})=0
$$

More recently, one of us investigated two-dimensional invariant sets in the context of the development of singularly perturbed systems or slow-fast systems. In particular, a new geometric approach has been developed in order to directly provide the slow invariant manifold of any $n$-dimensional dynamical system, singularly perturbed or not Ginoux, 2009. Thus flow curvature manifold can be defined as follows.

Theorem 2. The flow curvature manifold of a three-dimensional dynamical system is defined as the location of points where

$$
\kappa_{2}=0 \quad \Leftrightarrow \quad \phi(\boldsymbol{X})=\operatorname{Det}(\dot{\boldsymbol{X}}, \ddot{\boldsymbol{X}}, \dddot{\boldsymbol{X}})=0 .
$$

Solving this equation directly provides the analytical equation for the slow invariant manifold associated with such a system.

The flow curvature manifold is thus defined as Ginoux \& Letellier, 2009

$$
\phi(\boldsymbol{x})=\dot{\boldsymbol{X}} \cdot(\ddot{\boldsymbol{X}} \wedge \dddot{\boldsymbol{X}})=0 .
$$

Differentiating the acceleration vector $\ddot{\boldsymbol{X}}=\boldsymbol{J} \dot{\boldsymbol{X}}$ where $\boldsymbol{J}$ is the functional Jacobian matrix of the system, and inserting this expression into (9), we obtain

$$
\phi(\boldsymbol{X})=\underbrace{\dot{\boldsymbol{X}} \cdot(\mathcal{J} \dot{\boldsymbol{X}} \wedge \mathcal{J} \ddot{\boldsymbol{X}})}_{\phi_{c}}+\underbrace{\dot{\boldsymbol{X}} \cdot\left(\ddot{\boldsymbol{X}} \wedge \frac{\mathrm{d} \mathcal{J}}{\mathrm{d} t} \dot{\boldsymbol{X}}\right)}_{\phi_{t}}
$$

where $\phi_{c}$ is the time-independent component and $\phi_{t}$ is the time-dependent component. Since $\phi_{c}$ does not contain the time derivative of $\boldsymbol{J}$ it is associated with the linear component of the vector field and $\phi_{t}$ with the nonlinear 
component. In the neighborhood of fixed points $\boldsymbol{X}$, the time-independent component of the flow curvature manifold corresponds to the osculating plane. As a consequence, the attractor takes the shape of $\phi_{c}$ in this neighborhood because the osculating plane cannot be crossed by a trajectory Ginoux \& Letellier, 2009.

The two components of the flow curvature manifold of the memristive circuit (11) are shown in Fig. 3a. As expected, in the neighborhood of the fixed point, the time-independent component of the flow curvature manifold is tangent to the osculating plane. The component $\phi_{t}$ presents an hyperboloid (Fig. 3 b). The trajectory wraps around a significant part of this surface. Close to the fixed point, the trajectory crosses component $\phi_{t}$ as observed in the Rössler system Ginoux \& Letellier, 2009. Such an intersection between the trajectory and component $\phi_{t}$ could be an explaination for the limitation to the development of the dynamics.

\section{Topological analysis}

For $\alpha=0.98$, the memristor electronic circuit (10) produces a chaotic attractor (Fig. 2). A Poincaré section can be safely defined as

$$
\mathcal{P} \equiv\left\{x_{n}, z_{n} \mid y_{n}=0, \dot{y}<0\right\}
$$

Using parameter $\alpha$ as a bifurcation parameter, a bifurcation diagram is computed (Fig. 4). When $\alpha$ is increased, the diagram starts with a perioddoubling cascade as a route to chaos. A crisis is observed around $\alpha=$ 0.25507 , a value at which the attractor size suddenly increases. For slightly greater $\alpha$-values, a second period-doubling cascade issued from a period-2 orbit is observed. The development of the dynamics is observed at least up to $\alpha=0.53$. Then for $\alpha$ slightly less than 0.6 a bubbling is observed. The chaotic behavior is developed up to another bubbling observed in the middle of the period-3 window $(\alpha \approx 0.69)$. A last bubbling occurs in the middle of the second period-3 window ( $\alpha \approx 0.85)$. For greater $\alpha$-value, the dynamics is reduced by sub-critical bifurcations, destroying periodic orbits. The diagram is ended by an inverse period-doubling cascade leading to a period-1 limit cycle.

When $0.98<\alpha<1.276$, there are two attractors: one is chaotic and one is a period- 1 limit cycle. Depending on the initial conditions, the trajectory thus settles down onto one of these two attractors. This bistability disappears when the limit cycle collides with the chaotic attractor for $\alpha$ is 
slightly greater than 0.98 . We will thus investigate in details the topology of chaotic attractors in the neighborhood of these two crises.

Let us start with the chaotic attractor observed when the $\alpha$-value is close to the boundary crisis, that is, when $\alpha=0.98$ (Fig. 2). A first-return map to the Poincaré section (11) is computed (Fig. [5). It is a unimodal map with a differential maximum as expected after a period-doubling cascade. Such a maximum defines a partition of the attractor that allows us to distinguish two topologically distinct domains in the attractor Letellier et al, 1995. Increasing (decreasing) branches are necessarily associated with domains stripes - with an odd (even) number of $\pi$-twists. In the present case, the attractor is made of one stripe with one negative (anti-clockwise) and $\pi$-twist and one stripe with two negative $\pi$-twists as detailed below. We choose to label the increasing monotonic branch of the first-return map by symbol "2" and the decreasing monotonic branch by symbol "1". Each revolution over the attractor — around the fixed point — is thus encoded according to

$$
\mid \begin{array}{ll}
1 & \text { if } x_{n}>x_{\max } \\
2 & \text { if } x_{n}<x_{\max }
\end{array}
$$

where $x_{\max }=2.305$. Using this encoding, a trajectory in the phase space $\mathbb{R}^{3}(x, y, z)$ is mapped into a symbolic sequence Letellier et al, 1995.

The boundary crisis occurs when the symbolic dynamics is complete, that is, all symbolic sequences built with the two symbols "1" and "2" are realized as unstable periodic orbits. This always arises when the end of one of the monotonic branches touches the first bisecting line. In the present case (Fig. 5) this is the increasing branch that touches the bisecting line. The fact that a boundary crisis occurs exactly when the symbolic dynamics is complete is a rather common feature since most of the quadratic minimal systems investigated by Malasoma \& Letellier, 2010 exhibit such property.

The next step in the topological analysis is to extract the smallest unstable periodic orbits and to compute some linking numbers. A linking number counts the number of times a periodic orbit turns around another one. It can be counted in a regular plane projection by identifying orientated crossings. A crossing is counted only when one orbit "crosses" the other in the plane projection (self-crossings are ignored). Crossings are then orientated according to the third coordinates (see Letellier et al, 1995 for details). For instance, orbits (2) and (21) exhibit four negative crossings (Fig. 6). The corresponding linking number is thus

$$
\operatorname{Lk}(21,2)=\frac{1}{2}[-4]=-2 .
$$


This means that orbit (21) turns twice in the anti-clockwise direction around orbit (2).

We computed linking numbers for few pairs of periodic-orbits and checked that all of them were correctly predicted by the template shown in Fig. 77a). The template exhibits a global negative $\pi$-twist (left part of the template shown in Fig. (7a). As a consequence, the attractor is a non trivial suspension of a unimodal map (a trivial suspension would not present the global $\pi$-twist). The odd branches is thus in the middle of the attractor and not at its periphery. Due to that, it is said that the attractor is governed by an inverted Horseshoe map Gilmore, 1998. The template proposed in Fig. 7a can be deformed under an isotopy (a continuous deformation without any cutting) into a "standard" template according to [Tufillaro et al, 1992 . This standard representation allows us to describe the template in terms of the following linking matrix:

$$
M_{i j}=\left[\begin{array}{ll}
-1 & -2 \\
-2 & -2
\end{array}\right] .
$$

Diagonal terms $M_{i i}$ provides the numbers of (local) $\pi$-twist of each stripe. As shown in the standard template (Fig. $7 \mathrm{~b}$ ) there is therefore one negative $\pi$-twist for the odd - red - stripe (associated with the decreasing branch of the first-return map) and two negative $\pi$-twists for the even - green — strip (corresponding to the increasing branch). Off-diagonal terms $M_{i j}(i \neq j)$ means that stripe 1 crosses stripe 2 once in the negative way as observed in the right part of the template shown in Fig. 7b. With this convention, linking numbers can be algebraically predicted from the linking matrix (14) and the orbital sequences Le Sceller et al, 1994.

The second parameter value for which a topological analysis of the chaotic attractor solution to system (1) is chosen at the crisis observed around $\alpha=0.25507$. The chaotic attractor (Fig. 8) looks simpler than the attractor (Fig. 2) previously characterized. In particular, the global $\pi$ twist is no longer seen.

The first-return map to the Poincaré section (11) confirms that this is no longer an inverted Horseshoe since the increasing branch - associated with an even number of $\pi$-twists - is now in the middle of the attractor (lower $x$-value in Fig. 9, thus closer to the fixed point). This map has a "layered" decreasing branch. The end of the upper branch is exactly at the same ordinate $\left(x_{n+1}\right)$ as the differentiable maximum. This special characteristic was sometimes encountered in few other systems, and always associated with a crisis. This was for instance observed in a minimal system proposed by 
van der Schrier \& Maas, 2000]: in this case, a boundary crisis that ejects the trajectory to infinity was observed [Malasoma \& Letellier, 2010.

The symbolic dynamics is obviously not complete (the increasing branch does not touch the bisecting line) but as soon as the upper decreasing branch has an ordinate greater than the maximum, there is an abrupt creation of a large number of periodic orbits. Indeed, by increasing $\alpha$ to 0.25515 , the increasing branch is nearly completed and a third (increasing) branch occurs (Fig. 10), thus revealing the explosive character of the dynamics when the end of the upper decreasing branch has an ordinate that becomes greater than the ordinate of the maximum (Fig. 10).

Periodic orbits were extracted for $\alpha=0.25507$. Linking numbers were computed as shown in Fig. 11, All of the identified oriented crossings found were negative. As expected after the first sight of the chaotic attractor (Fig. 8), the global negative $\pi$-twist is no longer observed and all of the computed linking numbers were well predicted by the template proposed in Fig. 12 , This is now a trivial suspension of a unimodal map. The inner stripe close to the fixed point - has no local torsion and the outer stripe has a negative $\pi$ twist. The linking matrix is therefore as

$$
M_{i j}=\left[\begin{array}{cc}
0 & -1 \\
-1 & -1
\end{array}\right] \text {. }
$$

The cyan stripe associated with the increasing branch of the first-return map is thus encoded with integer " 0 " and the decreasing red branch with integer "1". Stripe 1 of this template is topologically equivalent to stripe 1 of the template proposed for $\alpha=0.98$ (Fig. 7b). In fact, this is the same stripe in both cases. When the bifurcation parameter $\alpha$ is varied from 0.25507 to 0.98 , periodic points in stripe 0 are progressively pruned while periodic points in stripe 2 are created.

At intermediate value like $\alpha=0.533$, the three branches co-exist (Fig. 13) in addition with a fourth one encoded by 4 . It can be shown in fact that the corresponding stripe present a local torsion equal to four negative $\pi$-twists. The chaotic attractor can be therefore split into four stripes whose topology can be synthetized by a template described by the linking matrix

$$
M_{i j}=\left[\begin{array}{cccc}
0 & -1 & -1 & -1 \\
-1 & -1 & -2 & -2 \\
-1 & -2 & -2 & -3 \\
-1 & -2 & -3 & -3
\end{array}\right] \text {. }
$$


This matrix matches with those proposed for the Rössler attractor of the funnel type [Letellier et al, 1995]. From the topological point of view, the dynamics produced by the memristive circuit is equivalent to those produced by the Rössler system.

The limitation of the dynamics could be explained by the time dependent component of the flow curvature manifold $\phi_{t}$ as we did for few Rössler-like systems in Ginoux \& Letellier, 2009. From component $\phi_{t}$ as shown in Fig. 15. it is obvious that the trajectory crosses component $\phi_{t}$ in the neighborhood of the fixed point. Such a feature was always remarked in different systems where some significant pruning was also noted. This means that, for instance, when four branches are observed in the first-return map (Fig. 13), all possible symbolic sequences are not realized as unstable periodic orbits. Since crossings of the trajectory occurs in the neighborhood of the fixed point and since this is the increasing branch encoded by 0 that is the closes to the fixed point, symbolic sequences with many consecutive " 0 " are forbidden. Consequently, the symoblic sequences containing these subsequences are no longer realized as periodic orbits. This is clearly seen in the four branches map (Fig. 13) where the increasing branch "0" is almost removed.

\section{Conclusion}

An electronic circuit with a memristor provides one of the simplest physical realization of a dynamical system producing chaos. The governing equations are not minimal from the algebraic point of view but this ensures in fact the robustness of the chaotic behaviour so produced. Indeed, it is very often observed that minimal systems have chaotic solutions for very tiny domain of their parameter space and the attraction basin is quite limited. The simplest memristive circuit is therefore a very good candidate for belonging to the class of benchmark physical realizations for producing chaotic behaviors.

In this paper we investigate the topological structure of phase portraits solution to this simple memristive circuit. It was shown that the chaotic attractors produced by this circuit were of the Rössler-like type, that is, they are topologically equivalent to the attractors produced by the wellknown Rössler system which results from a simplification of an oscillating abstract chemical reaction. The dynamical regimes solution to the simplest electronic circuit cannot be as developed in the Rössler system, due to a strong limitation of the development of the dynamics induced by the time dependent component of the flow curvature manifold. This leads to an 
inverse period-doubling cascade that constrains the behavior to be a limitcycle for large value of the bifurcation parameter.

\section{Acknowledgements}

L. Chua acknowledged support from the U.S. Air Force. AFORS grant no. ??.

Authors would like to thank Dr. Ubiratan Freitas who made the cover figure.

\section{References}

[Chua, 1971] Chua L. O. [1971] Memristor — the missing circuit element, IEEE Transaction on Circuit Theory 18, pp. 507-519.

[Chua, 1976 ] Chua L. O. \& Kang S. M. [1976] Memristive devices and systems, Proceedings of IEEE, 64, pp. 209-223.

[Ginoux, 2009] Ginoux J.-M. [2009] Differential geometry applied to dynamical systems, World Scientific Series on Nonlinear Science, Series A 66 (World Scientific, Singapore).

[Ginoux \& Letellier, 2009] Ginoux J.-M. \& Letellier C. [2009] Flow curvature manifolds for shaping chaotic attractors: I. Rössler-like systems, Journal of Physics A 42, 285101.

[Gilmore, 1998] Gilmore R. [1998] Topological analysis of chaotic dynamical systems, Reviews of Modern Physics, 70 (4), pp. 1455-1529.

[Gilmore et al, 2010] Gilmore R., Ginoux J.-M., Jones T., Letellier C. \& Freitas U. S. [2010] Connecting curves for dynamical systems, Journal of Physics A 43255101.

[Itoh \& Chua, 2008] Itoh M. \& Chua L. O. [2008] Memristor oscillators, International Journal of Bifurcation $E$ Chaos, 18 (11), pp. 3183-3206.

[Le Sceller et al, 1994] Le Sceller L., Letellier C. \& Gouesbet G. [1994] Algebraic evaluation of linking numbers of unstable periodic orbits in chaotic attractors, Physical Review E, 49 (5), pp. 4693-4695.

[Letellier et al, 1995] Letellier C., Dutertre P. \& Maheu B. [1995] Unstable periodic orbits and templates of the Rössler system: toward a systematic topological characterization, Chaos 5 (1), pp. 271-282. 
[Malasoma \& Letellier, 2010] Malasoma J.-M. \& Letellier C. in preparation,

[Muthuswamy \& Chua, 2010] Muthuswamy B. \& Chua L. O. [2010] Simplest chaotic circuit, International Journal of Bifurcation $\&$ Chaos, 20 (5), in press.

[Muthuswamy \& Kakate, 2010] Muthuswamy B. \& Kakate P. P. [2010] Memristor-based chaotic circuits, IETE Technical Review, 26 (6), pp. 417-429.

[Roth \& Peikert, 1998] Roth M. \& Peikert R. [1998] A higher-order method for finding vortex core lines, Proceedings IEEE of the Conference on Vizualization'98, pp. 143-150.

[Sprott, 1994] Sprott J. C., Some simple chaotic flows, Physical Review E 50 (2), pp. 647-650.

[Strukov et al, 2008] Strukov D. B., Snider G. S., Stewart D. R. \& Williams R. S. [2008] The missing memristor found, Nature, 453, 80-83.

[Thomas \& Kaufman, 2001] Thomas R. \& Kaufman M. [2001] Multistationarity, the basis of cell differentiation and memory: I. Structural conditions of multistationarity and other nontrivial behavior, Chaos, 11 (1), pp. $170-179$

[Tufillaro et al, 1992] Tufillaro N. B., Abbott T. \& Reilly J. [1992] An Experimental Approach to Nonlinear Dynamics and Chaos, (Addison-Wesley, New York).

[van der Schrier \& Maas, 2000] van der Schrier G. \& Maas L. R. M. [2000] The diffusionless Lorenz equations; Shil'nikov bifurcations and reduction to an explicit map, Physica D 141, pp. 19-36.

[Zhang \& Heidel, 1997] Zhang F. \& J. Heidel J. [1997] Non chaotic behaviour in three-dimensional quadratic systems, Nonlinearity 10, pp. 1289-1303. 

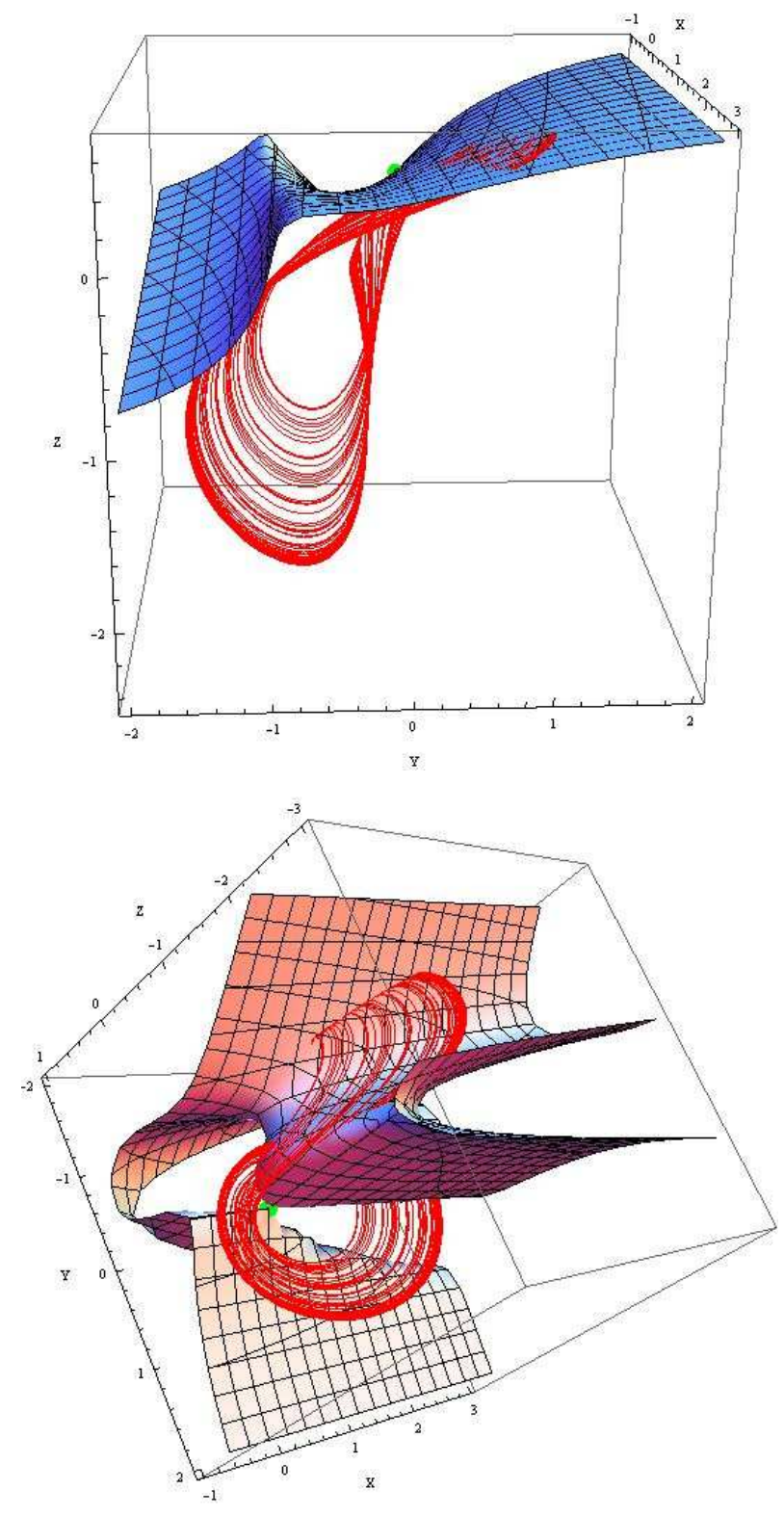

(a) Time-independent component $\phi_{c}$ (b) Time-dependent component $\phi_{t}$

Figure 3: The two components of the flow curvature manifold $\phi$ for the memristive circuit with parameter values: $\alpha=0.98$. 


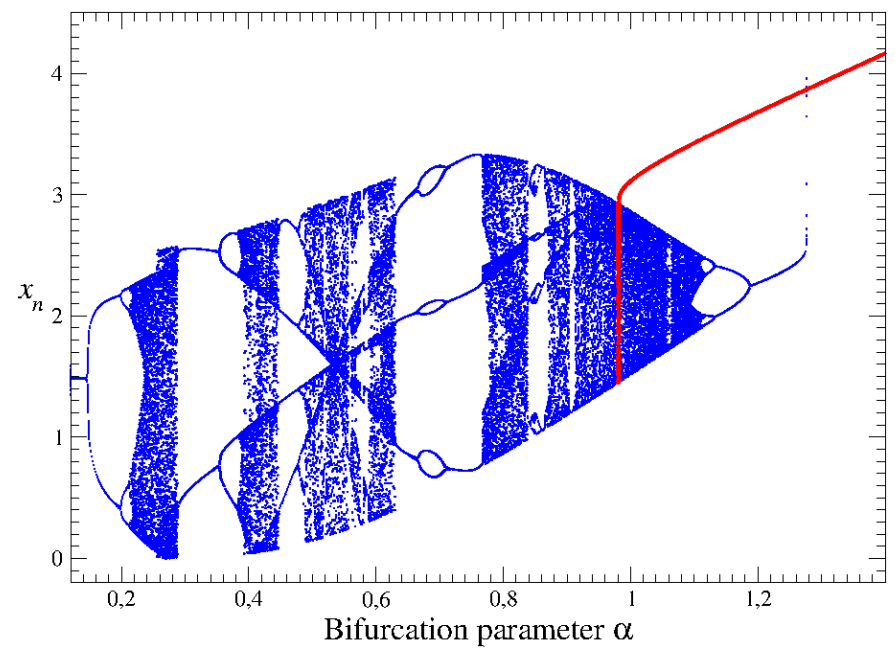

Figure 4: Bifurcation diagram versus parameter $\alpha$ of the minimal electronic circuit with a memristor.

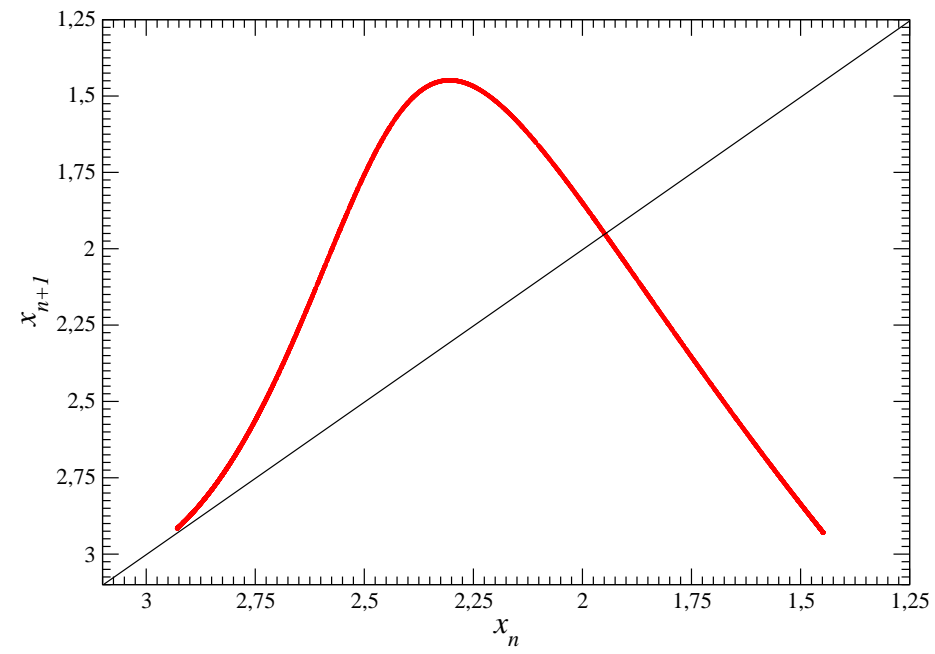

Figure 5: First-return map to a Poincaré section of the chaotic attractor solution to the minimal electronic circuit with a memristor. Parameter value: $\alpha=0.98$. 


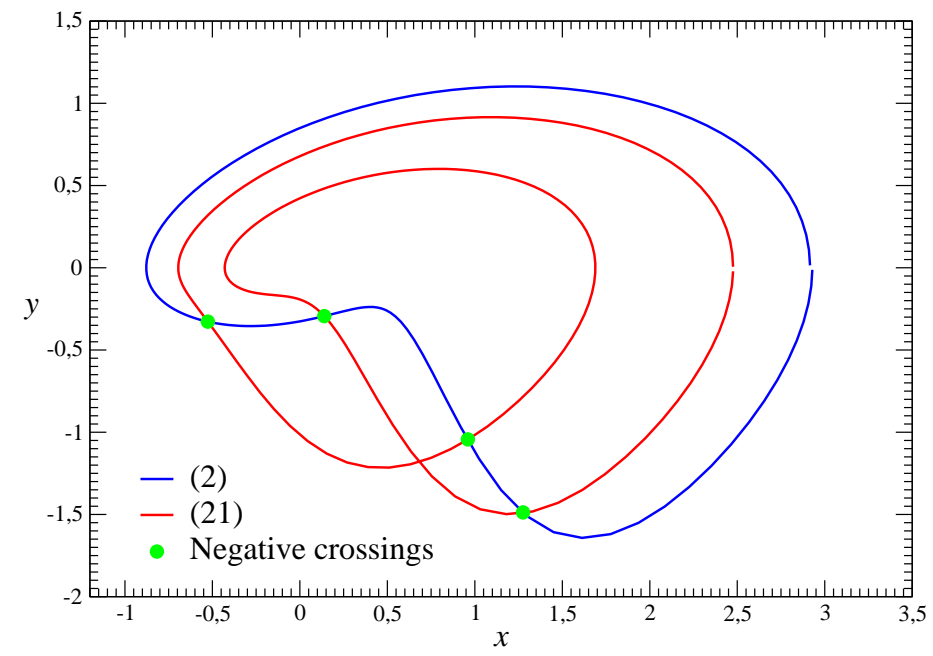

Figure 6: Pair of unstable periodic orbits extracted from the chaotic attractor solution to the simplest electronic circuit with a memristor. Parameter value: $\alpha=0.98$. The linking number $\operatorname{Lk}(2,21)=-2$ according to the four negative crossings identified in the $x-y$ plane projection. 


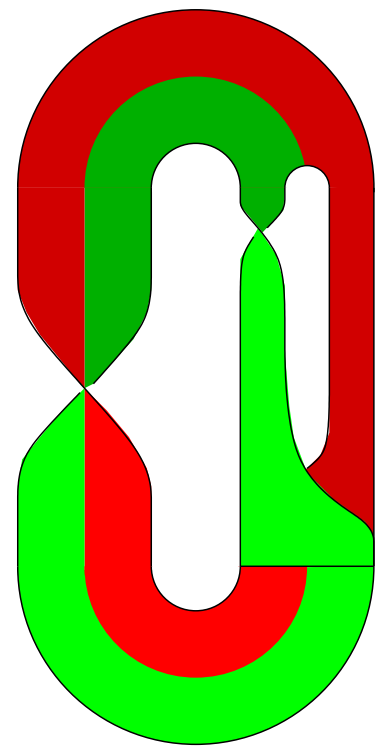

(a) Direct template

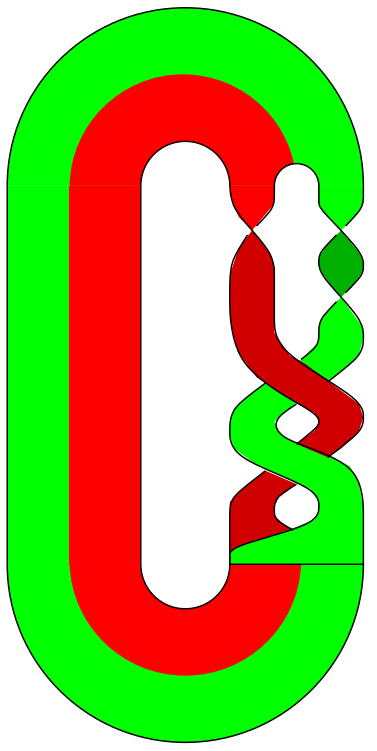

(b) Standard template

Figure 7: Template for the chaotic attractor solution to the minimal electronic circuit with a memristor. Parameter value: $\alpha=0.98$. The global negative $\pi$-twist (a) is send to the right part (b) under an isotopy.

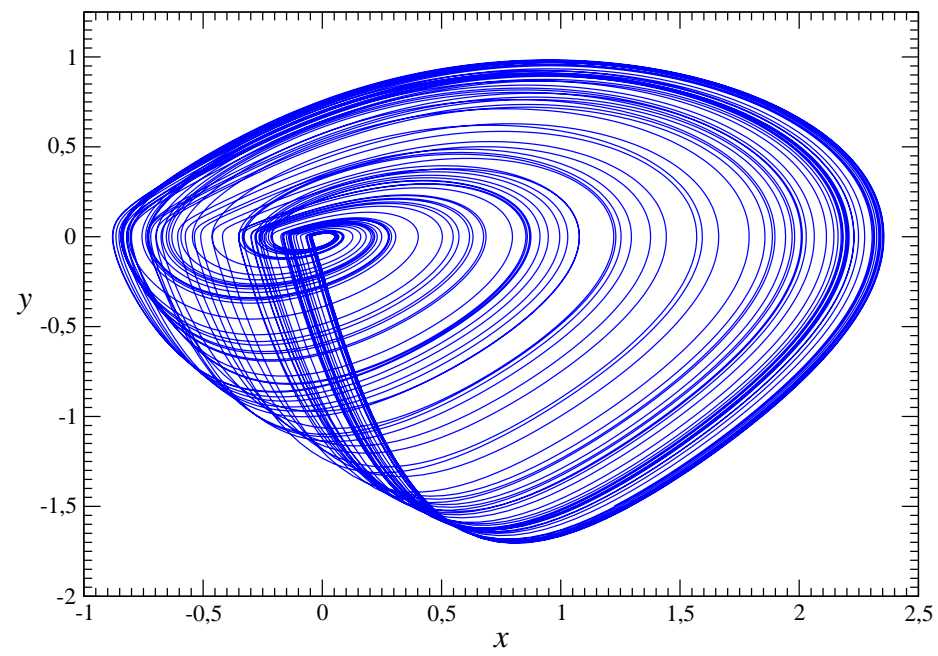

Figure 8: Chaotic attractor solution to the minimal electronic circuit with a memristor. Parameter value: $\alpha=0.25507$. 


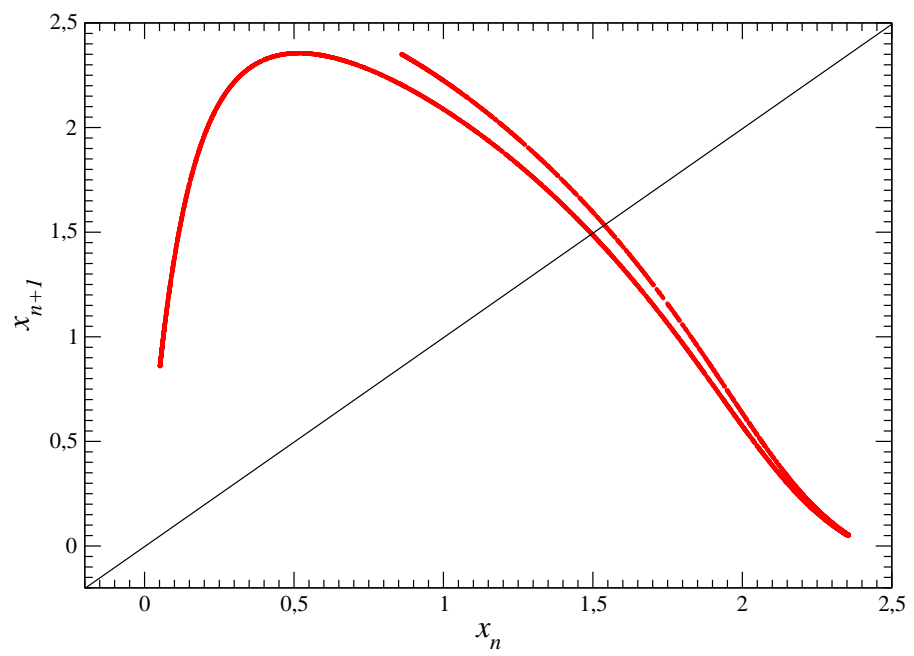

Figure 9: First-return map to a Poincaré section of the chaotic attractor solution to the minimal electronic circuit with a memristor. Parameter value: $\alpha=0.25507$.

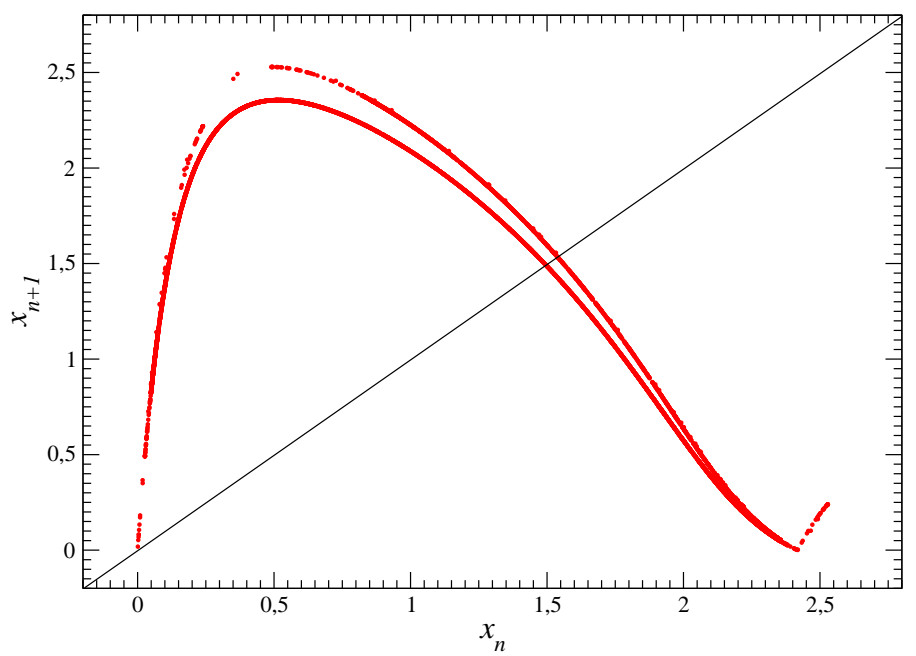

Figure 10: First-return map to a Poincaré section of the chaotic attractor solution to the minimal electronic circuit with a memristor. Parameter value: $\alpha=0.25515$. 


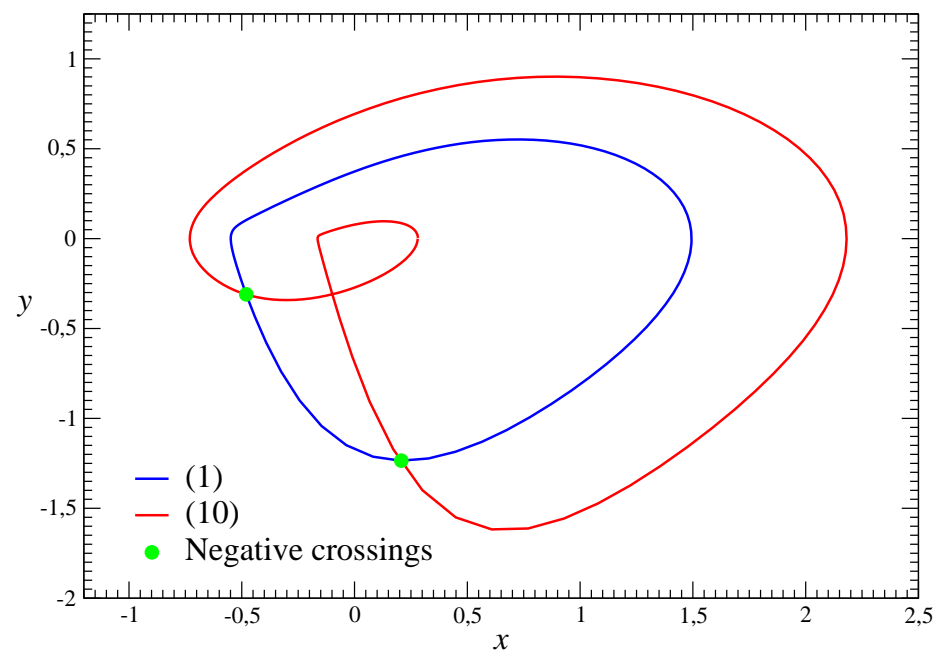

Figure 11: Pair of unstable periodic orbits extracted from the chaotic attractor solution to the minimal electronic circuit with a memristor. Parameter value: $\alpha=0.25507$. The linking number $\operatorname{Lk}(1,10)=-1$ according to the two negative crossings identified in the $x-y$ plane projection.

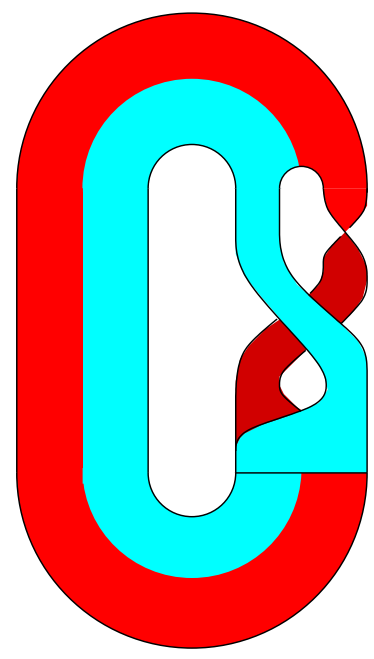

Figure 12: Template for the chaotic attractor solution to the minimal electronic circuit with a memristor. Parameter value: $\alpha=0.25507$. 


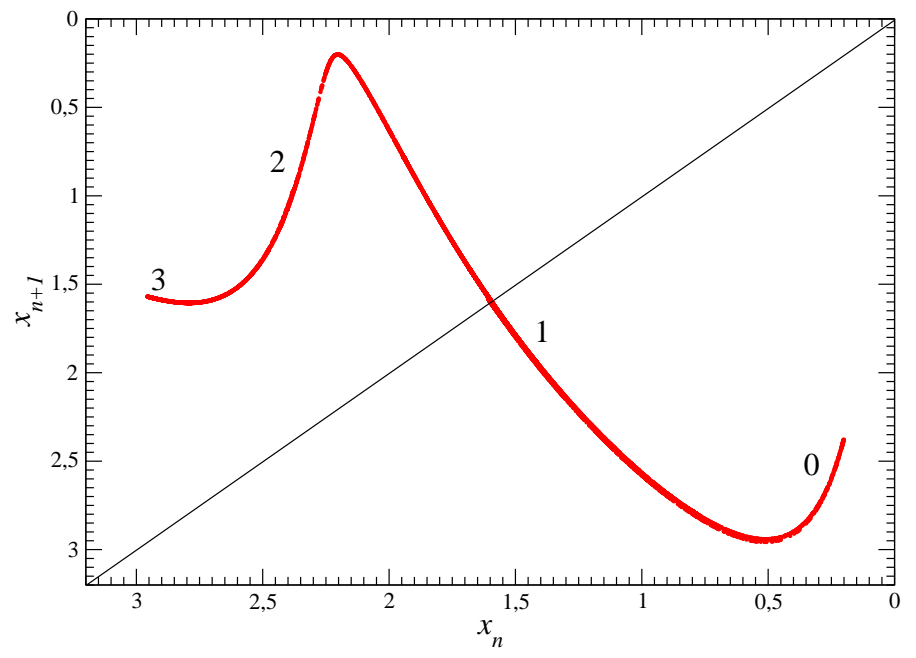

Figure 13: Three-modal first-return map to a Poincaré section of the chaotic attractor solution to system (1). Parameter values: $\alpha=0.533$.

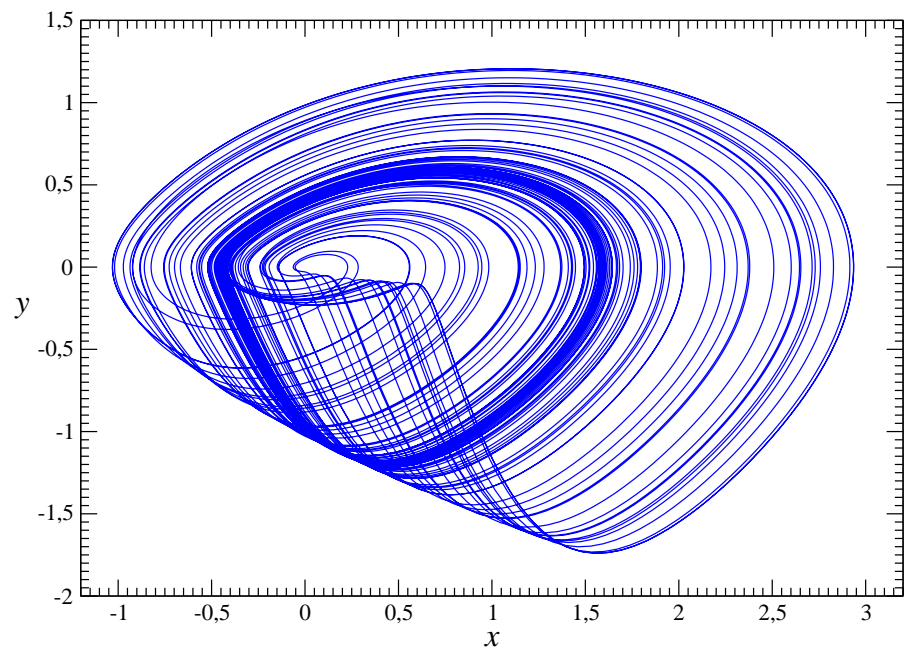

Figure 14: Chaotic attractor solution to system (1). Parameter values: $\alpha=0.533$. 


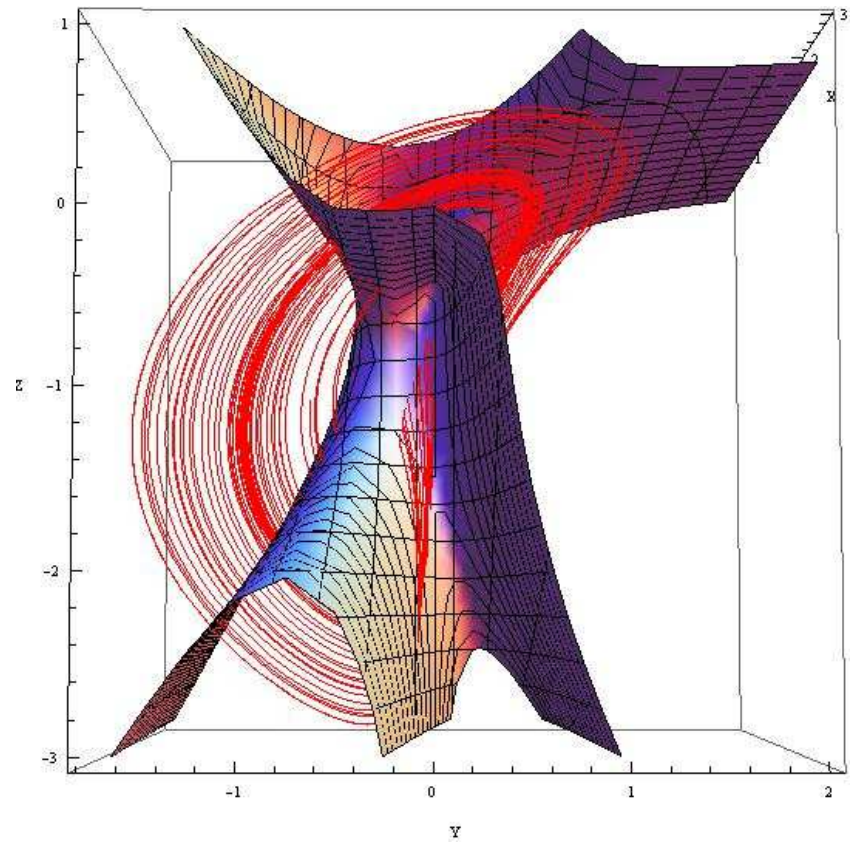

Figure 15: Time dependent component $\phi_{t}$ of the flow curvature manifold for the memristive circuit. Parameter value: $\alpha=0.533$. 
